\title{
Etude sur le Lben marocain
}

\author{
A. TANTAOUI-ELARAKI, M. BERRADA, A. EL MARRAKCHI \\ et A. BERRAMOU
}

\section{Ré s u m é}

Nous avons étudié l'évolution de certains groupes microbiens importants au cours de l'élaboration du Lben traditionnel marocain : flore mésophile aérobie totale, bactéries lactiques (Streptococci, Leuconostoc, Lactobacilli), coliformes, streptocoques fécaux, levures et moisissures. Parallèlement, le taux de matière grasse, l'extrait sec, l'acidité Dornic et le $\mathrm{pH}$ ont été déterminés.

Les streptocoques lactiques et les Leuconostoc sont les principaux groupes responsables de l'acidification du lait au cours de sa transformation en Lben. Les espèces les plus importantes sont Streptococcus lactis, S. diacetylactis, Leuconostoc lactis et L. cremoris. Les lactobacilles, très faiblement représentés, ne semblent pas jouer un rôle important dans l'élaboration du Lben. Les levures et les moisissures se développent régulièrement, notamment avec l'acidification du produit mais leur nombre n'atteint jamais un niveau très élevé. Cependant, elles pourraient participer à la production d'arôme.

Les coliformes et les streptocoques fécaux sont présents dans tous les échantillons.

Mots clés :

Lben - Microbiologie - Chimie - Technologie.

Titre abrégé : Lben marocain.

\section{S um $\mathrm{m}$ a ry}

\section{STUDY ON MOROCCAN LBEN}

The evolution of some important microbial groups during traditional Lben processing was studied. These groups were: the total mesophilic aerobic flora, the lactic acid bacteria (Streptoccocci, Leuconostoc, Lactobacilli), the Coliforms, the fecal Streptocacci,

Institut Agronomique et Vétérinaire Hassan II, B.P. 6202 Rabat-Instituts, Rabat (Maroc). 
Yeasts and Moulds. The fat content, the dry matter, the acidity and the $\mathrm{pH}$ of the same samples were also determined.

The results obtained showed that the lactic acid Streptococci and the Leuconostoc are the main groups responsible for the acidification of milk to be transformed into Lben. The most important species were Streptococcus lactis, S. diacetylactis, Leuconostoc lactis and L. cremoris. It was observed that the Lactobacilli were very weakly represented, so they seem not to take any prominent part in the transformation of milk into Lben. Yeasts and Moulds grew regularly, especially when the product became acid, but their number never reached a high level in the present study. However they would participate in the formation of the Lben aroma. Coliforms and fecal Streptococci were present in all the samples.

Keys words:

Lben - Microbiology - Chemistry - Technology.

\section{INTRODUCTION}

Le Lben marocain est une boisson préparée par fermentation spontanée du lait cru jusqu'à coagulation, suivie d'un léger mouillage, puis d'un barattage, permettant de recueillir une part plus ou moins importante de matière grasse sous forme de beurre dit « beldi ".

Sa préparation, très simple, est demeurée au stade familial ou artisanal : le lait est abandonné à lui-même dans une jarre en terre cuite ou une outre en peau de chèvre jusqu'à sa coagulation. Celle-ci se fait à température ambiante et dure 24 à 48 h suivant la saison. Le barattage qui lui succède est réalisé soit dans l'outre, qu'un manipulateur doit secouer énergiquement avec les deux mains (photo 1), soit dans une jarre, en utilisant un instrument constitué d'un manche long portant à son extrêmité inférieure deux disques en bois de diamètres différents (photo 2). Dans un cas comme dans l'autre, cette opération dure 30 à $40 \mathrm{~min}$. A la fin du barattage, on ajoute généralement un certain volume d'eau (environ $10 \%$ du volume du lait), chaude ou froide, suivant la température ambiante, de façon à ramener la température de l'ensemble à un niveau convenable au rassemblement des grains de beurre. Celui-ci est récupéré, généralement à la main, mais certains fabriquants filtrent le Lben sur une toile, dans le but de recueillir le maximum de beurre " beldi ", produit de grande valeur marchande.

L'objet du présent travail est de déterminer les caractéristiques microbiologiques et physico-chimiques du Lben marocain en vue, essentiellement, de connaître les espèces microbiennes responsables de l'élaboration du produit. Pour ce faire, nous avons analysé 20 échantillons du commerce, et procédé nous-mêmes à la préparation, au laboratoire, de 6 échantillons sur lesquels nous avons suivi l'évo- 


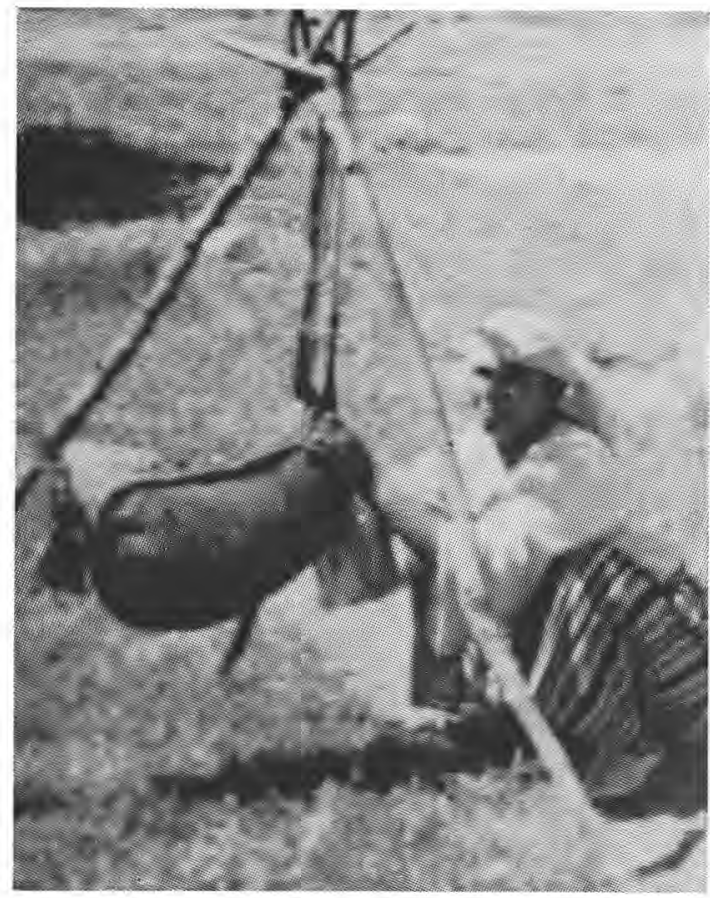

Photo 1

Outre en peau de chèvre pour la fermentation et le barattage du Lben (photo due à l'obligeance de $\mathrm{M}$. Moussa Ettalibi).

Goatskin for acidifying and churning of Lben (picture due to the courtery of $\mathrm{Mr}$ Moussa Ettalibi).

lution physico-chimique et microbiologique depuis la matière première (lait) jusqu'au produit fini (Lben).

Nous avons donc, volontairement, donné la priorité aux espèces d'intérêt technologique, laissant de côté, pour le moment la flore de contamination.

\section{MATERIEL ET METHODES}

\subsection{Préparation du Lben au laboratoire}

Du lait cru de grand mélange, provenant d'une étable de 135 vaches, est placé dans une jarre en terre cuite préalablement nettoyée avec de l'eau savonneuse, puis désinfectée à l'eau javellisée, et rincée à l'eau chaude $\left(70^{\circ} \mathrm{C}\right)$. Après $44 \mathrm{~h}$ à $20-22^{\circ} \mathrm{C}$, le lait coagulé est transvasé dans une jarre de barattage où cette opération s'effectue comme indiqué précédemment. On ajoute un volume d'eau équivalent à $10 \%$ du volume de lait traité, afin de favoriser le rassemblement des grains de beurre. Celui-ci est recueilli avec une louche métallique flambée à l'alcool. 


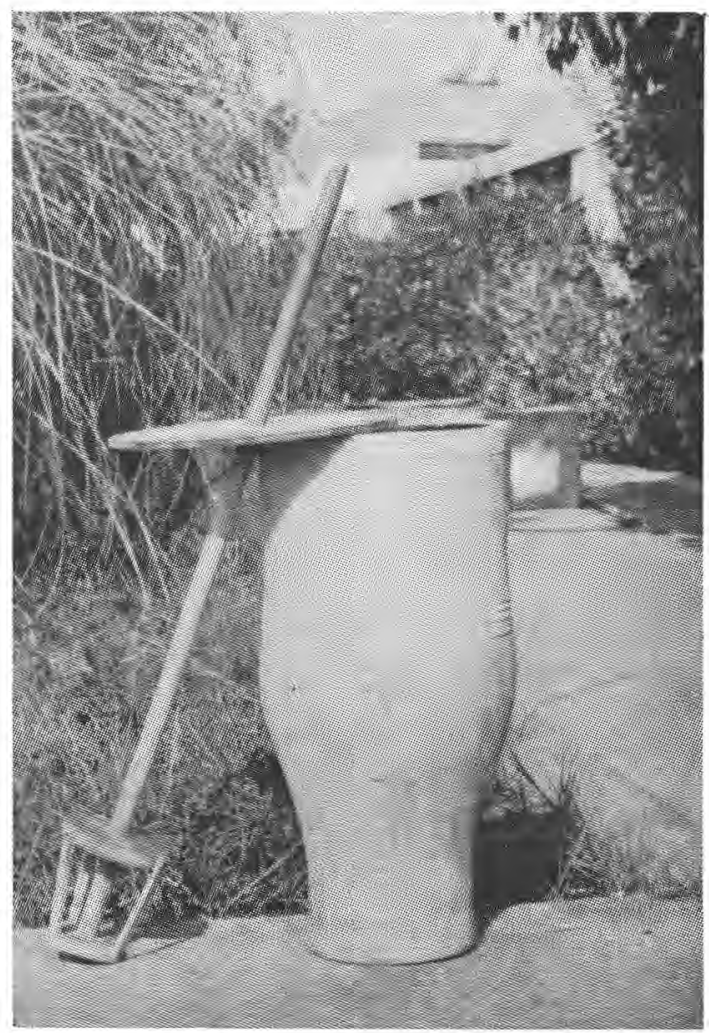

Photo 2

Jarre en terre cuite pour la fermentation du Lben, et instrument en bois utilisé pour le barattage du produit dans la jarre.

Earthenwar jar for Lben fermentation and wooden tool used for churning.

\subsection{Analyses physico-chimiques et microbiologiques}

Les caractéristiques physico-chimiques ont été déterminées sur les 20 échantillons de Lben du commerce, prélevés au hasard sur le marché de Rabat.

D'autre part, sur les 6 fabrications effectuées au laboratoire, l'évolution des caractères physico-chimiques et microbiologiques a été suivie depuis le lait frais $(0 \mathrm{~h})$ jusqu'au produit fini $(44 \mathrm{~h})$ en effectuant des prélèvements intermédiaires 16 h, 18 h, 20 h, 22 h, 24 h, $26 \mathrm{~h}$ et $40 \mathrm{~h}$ après la traite.

\subsubsection{ANALYSES PHYSICO-CHIMIQUUES}

- le $\mathrm{pH}$ a été déterminé à 0,1 unité près à l'aide d'un $\mathrm{pH}$ mètre Orion modèle 301 ;

- l'acidité titrable a été mesurée par neutralisation à l'aide d'une solution N/9 de soude, et exprimée en degrés Dornic; 
- la détermination de l'extrait sec a été effectuée par la méthode de référence préconisée par la Fédération Internationale de Laiterie (1962);

- enfin, la matière grasse a été dosée par la méthode acidobutyrométrique de Gerber.

\subsubsection{Analyses Microbiologiques}

a) Numération des micro-organismes

Après dilution des échantillons dans une solution de Ringer au $1 / 4$ stérile, nous avons procédé au dénombrement des différentes catégories de micro-organismes mentionnées au tableau 1, qui indique également les milieux de culture utilisés et les conditions d'incubation.

\section{b) Identification}

- L'identification des streptocoques lactiques et des Leuconostoc a été réalisée en se basant sur les caractères morphologiques, biochimiques, et culturaux préconisés par Girard et Rougieux (1967), Buttiaux et al. (1974), le Bergey's Manual of Determinative Bacteriology (1974), Harrigan et Mc Cance (1976) et Sharpe (1979) (tab. 2).

Cette étude a porté sur 77 souches prélevées au hasard au cours des différents stades de fabrication, soit 47 streptocoques et $30 \mathrm{Leu}$ conostoc, isolés de leurs milieux respectifs de dénombrement.

Les résultats de cette identification ont été ensuite vérifiés sur 11 souches en employant le système API/5OL mis au point spécialement pour l'identification des bactéries de la famille des Lactobacillaceae (nomenclature de la 8 e édition du "Bergey's Manual »).

- Pour les levures, 22 souches ont été identifiées sur la base des critères préconisés par Girard et Rougieux (1967), Lodder (1971) et Barnett et al. (1979) (tab. 2).

- Enfin, les moisissures ont été identifiées par observation de l'aspect des thalles sur milieu de Sabouraud, et examen microscopique à l'état frais.

\section{RESULTATS}

\subsection{Analyses physico-chimiques}

\subsubsection{CARACTÉRISTIQUES PHYSICO-ChimiQues DU LBEN}

Les Lbens du commerce analysés présentent en moyenne un $\mathrm{pH}$ de 4,4 et une acidité de $75^{\circ}$ Dornic, leur taux de matière grasse moyen est de 9,6 g/l pour un extrait sec de 87,9 g/l (tab. 3).

Quant aux Lbens fabriqués au laboratoire, s'ils présentent à peu près la même acidité et le même $\mathrm{pH}$ moyens que les échantillons 
TABLEAU 1 - TABLE 1

Numération des micro-organismes du Lben : milieux utilisés et conditions d'incubation

Enumération of microorganisms from Lben: used mediums and incubation conditions

\begin{tabular}{|c|c|c|c|c|}
\hline \multirow{2}{*}{ Groupes de micro-organismes } & \multirow{2}{*}{ Milieux de dénombrement } & \multicolumn{2}{|c|}{ Incubation } & \multirow{2}{*}{ Observations } \\
\hline & & $\begin{array}{l}\text { Température } \\
\left({ }^{\circ} \mathrm{C}\right)\end{array}$ & Durée & \\
\hline Flore mésophile aérobie totale & $\begin{array}{l}\text { gélose pour numération } \\
\text { (Institut Pasteur 6447) }\end{array}$ & 30 & $3 \mathrm{j}$ & \\
\hline Streptocoques lactiques & Elliker et Anderson (a) & 30 & $3 j$ & \\
\hline Leuconostoc & milieu hypersaccharosé (b) & 25 & $3-8 \mathrm{j}$ & \\
\hline Lactobacilles & MRS (Oxoïd CM 361) & 30 & $24-36 \mathrm{~h}$ & $\begin{array}{l}\text { anaérobiose } \\
\text { (jarre à anaérobies) }\end{array}$ \\
\hline Levures et moisissures & $\begin{array}{l}\text { Sabouraud (Oxoïd CM } 147 \text {, } \\
\text { gélosé à } 1,2 \%)\end{array}$ & 30 & $3 \mathrm{j}$ & \\
\hline Coliformes & gélosé au désoxycholate & 37 & $24 \mathrm{~h}$ & double couche \\
\hline Streptocoques fécaux & $\begin{array}{l}\text { Litsky liquide } \\
\text { (Institut Pasteur 6486) }\end{array}$ & 37 & $24-48 \mathrm{~h}$ & (c) \\
\hline \multicolumn{2}{|c|}{$\begin{array}{l}\text { (a) Milieu d'Elliker et Anderson : Tryptone Difco } 20 \mathrm{~g} \text {; } \\
\text { extrait de levure Difco } 5 \mathrm{~g} \text {; glucose } 5 \mathrm{~g} \text {; lactose } 5 \mathrm{~g} \text {; } \\
\text { gélatine } 2,5 \mathrm{~g} \text {; chlorure de sodium } 4 \mathrm{~g} \text { : Agar Difco } \\
15 \mathrm{~g} \text { : eau distillée } 1000 \mathrm{ml}-\mathrm{pH} 6,8 .\end{array}$} & \multicolumn{3}{|c|}{$\begin{array}{l}150 \mathrm{~g} \text {; phosphate dipotassique } 2 \mathrm{~g} \text {; chlorure de sodium } \\
1 \mathrm{~g} \text {; sulfate de magnésium à } 7 \mathrm{molécules} \mathrm{d'eau} 0,2 \mathrm{~g} \text {; } \\
\text { Agar } 15 \mathrm{~g} \text {; eau distillée } 1000 \mathrm{ml}-\mathrm{pH} 6,8 \text {. }\end{array}$} \\
\hline \multicolumn{2}{|c|}{$\begin{array}{l}\text { Milieu hypersaccharosé : extrait de viande } 10 \mathrm{~g} \text {; extrait } \\
\text { de levure } 3 \mathrm{~g} ; \text { Bactocasitone Difco } 2,5 \mathrm{~g} \text {; saccharose }\end{array}$} & \multicolumn{3}{|c|}{$\begin{array}{l}\text { Echantillon chauffé à } 60^{\circ} \mathrm{C} \text { pendant } 30 \mathrm{~min} \text {. Test pré- } \\
\text { somptif sur milieu de Rothe (Institut Pasteur 6490). }\end{array}$} \\
\hline
\end{tabular}


TABLEAU 2 - TABLE 2

Critères employés pour l'identification des bactéries lactiques et des levures isolées du Lben

Criteria used for identification of lactic bacteria and yeasts isolated from Lben

\begin{tabular}{|c|c|c|c|}
\hline Critères & $\begin{array}{l}\text { Streptocoques } \\
\text { et } \\
\text { Leuconostoc }\end{array}$ & Levures & Observations \\
\hline Morphologie cellulaire & $\times$ & $\times$ & \\
\hline Coloration de Gram & $x$ & & \\
\hline Aspect des colonies & & $x$ & Sur milieu de Sabouraud (Oxoïd CM 147) gélosé. \\
\hline Aspect en milieu liquide & & $x$ & Sur bouillon à l'extrait de malt. \\
\hline $\begin{array}{l}\text { Croissance à } 10^{\circ} \mathrm{C} \\
\text { Croissance à } 37^{\circ} \mathrm{C} \\
\text { Croissance à } 45^{\circ} \mathrm{C}\end{array}$ & $\begin{array}{l}\times \\
\times \\
\times\end{array}$ & & $\begin{array}{l}\text { Sur bouillon ordinaire à } 1 \% \text { de glucose et } 0,3 \% \text { d'extraits } \\
\text { de levures Difco. }\end{array}$ \\
\hline $\begin{array}{l}\text { Résistance au sel à } 2 \text { \% } \\
\text { Résistance au sel à } 4 \\
\text { Résistance au sel à } 6,5 \%\end{array}$ & $\begin{array}{l}\times \\
\times \\
\times\end{array}$ & & $\begin{array}{l}\text { Sur bouillon contenant : lactose } 0,5 \% \text {, tryptone } 0,5 \% \text {, } \\
\text { lablemco } 0,5 \% \text {. }\end{array}$ \\
\hline Recherche de la catalase & $\times$ & & Eau oxygénée à 10 volumes. \\
\hline $\mathrm{CO}_{2}$ à partir du glucose & $x$ & & $\begin{array}{l}\text { Extrait de viande } 0,5 \mathrm{~g} \text {, tryptone } 1 \mathrm{~g} \text {, peptone } 0,5 \mathrm{~g} \text {, glucose } \\
5 \mathrm{~g} \text {, chlorure de sodium } 0,5 \mathrm{~g} \text {, gélatine } 15 \mathrm{~g} \text {, eau } 100 \mathrm{ml}, \mathrm{pH} 7 \text {. }\end{array}$ \\
\hline Production d'acétoïne & $x$ & & Culture sur lait et réaction de Voges-Proskauer. \\
\hline
\end{tabular}


TABLEAU 2 - TABLE 2 (suite)

Action sur lait tournesolé

Désamination de l'arginine

Assimilation des sucres :

glucose

saccharose

maltose

lactose

raffinose

Fermentation des sucres :

glucose

fructose

maltose

lactose

saccharose

raffinose

mannitol

sorbitol

Observations

$$
\text { et }
$$

Leuconostoc

$\times$

$\times$

Extrait de levure $2,5 \mathrm{~g}$, tryptone $5 \mathrm{~g}$, phosphate dipotassique $2 \mathrm{~g}$, glucose $0,5 \mathrm{~g}$, arginine-L-monohydrate $3 \mathrm{~g}$, eau $1000 \mathrm{ml}$, pH 7.

Sur milieu Bacto Yeast Nitrogen Base (Difco ${ }^{\circ}$ 392) additionné des sucres à $0,5 \%$ sauf pour le raffinose $(1 \%)$.

\section{$x$}

$x$

$x$

$x$

- Pour les streptocoques et Leuconostoc : peptone $5 \mathrm{~g}$, extrait de viande $5 \mathrm{~g}$, tryptone $10 \mathrm{~g}$, pourpre de bromocrésol $0,002 \mathrm{~g}$, sucre à tester $5 \mathrm{~g}$, eau distillée $1000 \mathrm{ml}$.

- Pour les levures : eau de levure additionnée de $2 \%$ du sucre à tester. 
TABLEAU 3 - TABLE 3

Caractéristiques physico-chimiques du Lben du commerce et du Lben fabriqué au laboratoire

Physico-chemical characteristics of commercial Lben and laboratory made Lben

\begin{tabular}{|c|c|c|c|c|c|c|}
\hline & \multicolumn{3}{|c|}{ Lben du commerce (20 échantillons) } & \multicolumn{3}{|c|}{ Lben fabriqué (6 échantillons) } \\
\hline & Minimum & Moyenne & Maximum & Minimum & Moyenne & Maximum \\
\hline $\mathrm{pH}$ & 3,9 & 4,4 & 4,8 & 4,3 & 4,4 & 4,4 \\
\hline Acidité $\left({ }^{\circ}\right.$ Dornic $)$ & 70 & 75 & 82 & 71 & 74,5 & 78 \\
\hline Matière grasse $(\mathrm{g} / \mathrm{l})$ & 4 & 9,6 & 15 & 10 & 11,8 & 14 \\
\hline Extrait $\sec (\mathrm{g} / \mathrm{l})$ & 77,2 & 87,9 & 102,7 & 92,5 & 96,2 & 100,8 \\
\hline
\end{tabular}




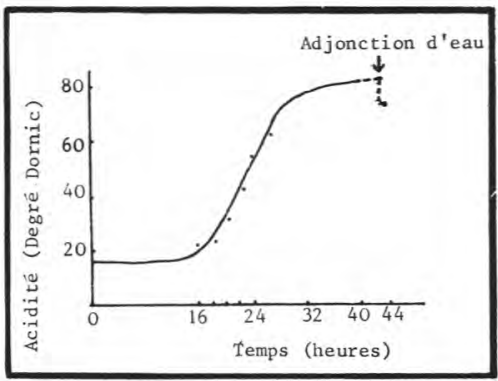

fig. 1

Evolution de l'acidité Dornic au cours de l'élaboration du Lben (moyennes des déterminations relatives à 6 échantillons).

Development of Dornic acidity during Lben making (average of 6 determinations).

du commerce, ils sont plus riches en matière grasse et en extrait $\sec (11,8$ et $96,2 \mathrm{~g} / \mathrm{l}$, respectivement).

Le tableau 3 permet également d'observer une plus grande variabilité des caractéristiques physico-chimiques des produits du commerce par rapport à ceux fabriqués par nous-mêmes. Ceci est particulièrement net pour la matière grasse (qui varie de 4 à $15 \mathrm{~g} / \mathrm{l}$ ) et l'extrait $\sec (77,2$ à $102,7 \mathrm{~g} / 1)$.

\subsubsection{Evolution DE L'ACIDITÉ EN FONCTION DU TEMPS}

La figure 1 montre l'évolution de l'acidité, exprimée en degrés Dornic, en fonction du temps. Les valeurs portées sur la courbe représentent les moyennes des 6 résultats relatifs aux Lbens fabriqués au laboratoire. On note qu'après une phase de latence de 12 à $16 \mathrm{~h}$, l'acidité augmente rapidement, puis cette évolution se ralentit 26 à $30 \mathrm{~h}$ après la traite, pour chuter ensuite brusquement suite à l'adjonction d'eau à la fin du processus de fabrication.

\subsection{Analyses microbiologiques}

\subsubsection{ETUde QUANTITATIVE}

a) Flore microbienne du Lben

Nous avons dénombré les différentes catégories de germes mentionnés au tableau 1 dans le lait frais et à différents stades de la fabrication jusqu'au produit fini. Le tableau 4 résume les résultats obtenus pour le lait et le Lben, indépendamment de l'évolution qui se produit en cours de fabrication. 
TABLEAU 4 - TABLE 4

Flore microbienne du lait et du Lben (6 échantillons)

Microbial flora of milk and Lben (6 samples)

\begin{tabular}{|c|c|c|c|c|c|c|}
\hline & \multicolumn{3}{|c|}{ Lait frais } & \multicolumn{3}{|c|}{ Lben } \\
\hline & Minimum & Moyenne & Maximum & Minimum & Moyenne & Maximum \\
\hline Flore mésophile aérobie totale $\left(\times 10^{5} / \mathrm{ml}\right)$ & 0,74 & 400 & 22700 & 410 & 21500 & 38000 \\
\hline Streptocoques lactiques $(\times 105 / \mathrm{ml})$ & 0,27 & 2,9 & 12,3 & 5,9 & 7600 & 15300 \\
\hline Leuconostoc $\left(\times 10^{4} / \mathrm{ml}\right)$ & 0,35 & 1,43 & 6,1 & 4,2 & 167 & 610 \\
\hline Lactobacilles & - & - & - & + & + & + \\
\hline Levures et moisissures $\left(\times 10^{2} / \mathrm{ml}\right)$ & - & - & - & 3,5 & 8,5 & 10,8 \\
\hline Coliformes $\left(\times 10^{3} / \mathrm{ml}\right)$ & 0,48 & 1,8 & 6,7 & 43 & 50,5 & 85 \\
\hline Streptocoques fécaux $\left(\times 10^{3} / \mathrm{ml}\right)$ & 2,5 & 3,8 & 11 & 6 & 101,6 & 450 \\
\hline
\end{tabular}


Il est très net que, parmi les germes que nous avons recherchés, les streptocoques lactiques forment le groupe le plus important dans le Lben. Leur nombre se trouve multiplié par 2600 entre le début et la fin de l'élaboration.

Aucun autre groupe ne marque un développement aussi important, on note cependant une augmentation de toutes les catégories.

Il est à noter que les lactobacilles n'ont jamais pu être mis en évidence dans le lait, et que leur nombre, même dans le Lben, a toujours été trop faible (1 à 8 colonies par boîte de Petri) pour qu'on pût les quantifier avec précision.

Les levures et moisissures, également non détectées dans le lait, apparaissent très nettement dans le produit fini où elles sont représentées à raison de 850 germes/ml en moyenne.

b) Evolution de la microflore au cours de l'élaboration du Lben

La figure 2 montre l'évolution observée dans le cas de l'échantillon 4 qui nous a semblé le plus représentatif, cela pour la flore

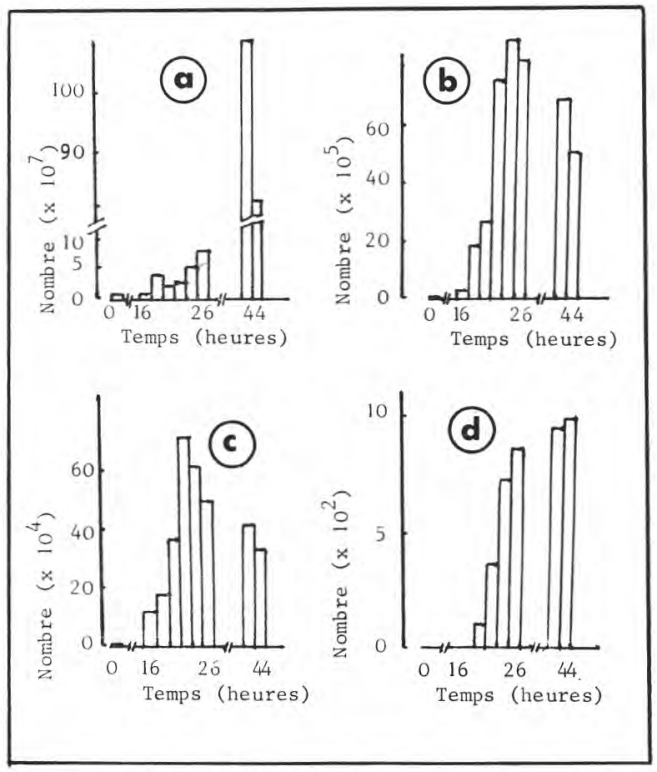

fig. 2

Evolution des groupes microbiens importants au cours de l'élaboration du Lben ; a : flore mésophile aérobie totale ; b : streptocoques lactiques ; c : Leuconostoc ; d : levures et moisissures.

Evolution of main microbial groups during Lben making; a : total mesophilic aerobic flora ; $b$ : lactic streptococci ; $c$ : Leuconostoc ; $d:$ yeasts and fungi. 
mésophile aérobie totale, les streptocoques lactiques, les Leuconostoc et les levures et moisissures. En effet, dans tous les cas :

- la flore mésophile aérobie totale augmente régulièrement entre le lait frais et la phase finale d'élaboration, après quoi elle diminue, par suite de l'adjonction d'eau;

- les streptocoques lactiques et les Leuconostoc évoluent également de façon régulière jusqu'à un certain niveau, variable d'un échantillon à l'autre, puis leur nombre décroît. Il ne semble pas y avoir de relation entre l'évolution des Leuconostoc et celle des streptocoques lactiques;

- les levures et spores de moisissures n'apparaissent que 20 à $22 \mathrm{~h}$ après la traite, et leur nombre augmente alors régulièrement jusqu'au produit fini ;

- enfin, pour ce qui est de l'évolution des coliformes, et des streptocoques fécaux, il est difficile de dégager une tendance générale à partir de nos résultats.

\subsubsection{Etude Qualitative : IDENTIFication}

a) Ferments lactiques

Nous avons pu identifier 32 souches de Streptococcus lactis et 15 de $S$. diacetylactis parmi les 47 isolements effectués à partir du milieu d'Elliker et Anderson. Quant aux 30 souches de Leuconostoc isolées à partir du milieu hypersaccharosé, elles se répartissent en 17 souches de L.cremoris et 13 de L.lactis.

Les résultats obtenus ont été confirmés par le système API/5OL sur 11 souches, prélevées au hasard, qui se répartissent comme suit : 5 Streptococcus lactis, 2 S.diacetylactis, 3 Leuconostoc lactis et 1 L.cremoris.

b) Levures

Les 22 souches de levures identifiées se répartissent en 16 représentants de Saccharomyces cerevisiae et 6 de Kluyveromyces marxianus.

c) Moisissures

Une espèce unique de moisissure a été trouvée : sur milieu solide, elle forme des thalles de couleur blanche à crème, de forme circulaire rayonnante et duveteuse. L'observation microscopique à l'état frais entre lame et lamelle révèle une structure caractéristique de Geotrichum candidum : ramification dichotomique, formation d'arthrospores cylindriques ou ovalaires, etc.

\section{DISCUSSION}

\subsection{Analyses physico-chimiques}

C'est dans les échantillons du commerce que l'on note les teneurs les plus faibles en matière grasse (certainement dues au souci des 
producteurs de récupérer le maximum de beurre) et les taux les plus bas d'extrait sec (liés à une plus forte adjonction d'eau), les deux phénomènes se traduisant par un profit plus grand pour le producteur au détriment de la valeur nutritionnelle du produit.

On peut également noter une variabilité plus grande de l'acidité et du $\mathrm{pH}$ des échantillons du commerce par rapport à ceux fabriqués au laboratoire.

\subsection{Analyses microbiologiques}

Tous les groupes microbiens étudiés connaissent un développement important au cours de l'élaboration du Lben, à l'exception des lactobacilles dont le nombre reste très faible.

Les streptocoques lactiques constituent la flore la plus abondante dans le Lben. Leur évolution se superpose à celle de l'acidité, jusqu'à un certain niveau, variable suivant les échantillons, à partir duquel on note un ralentissement, dû certainement à l'effet inhibiteur de l'acide lactique.

Les Leuconostoc suivent à peu près la même évolution, tout en restant beaucoup moins nombreux que les streptocoques.

Ces 2 catégories de germes jouent certainement un rôle prépondérant dans l'acidification et l'arômatisation du Lben. En effet, les souches que nous avons identifiées se répartissent entre des espèces homofermentaires acidifiantes ( $S$. lactis, et $S$. diacetylactis) et des espèces arômatisantes hétérofermentaires (Leuconostoc lactis et $L$. cremoris) ou homofermentaires (S. diacetylactis).

Notons que le Streptococcus cremoris n'a pas été détecté dans notre étude, contrairement aux résultats de Harrati (1974) relatifs au Lben algérien.

Le faible niveau des lactobacilles dans nos échantillons n'est pas facile à expliquer; Harrati (1974) avait noté leur absence totale dans le Lben algérien et émis les hypothèses suivantes :

- la fermentation du lait par les streptocoques ne serait pas suffisamment avancée pour permettre aux lactobacilles de se développer ;

- la présence de facteurs inhibiteurs pour les lactobacilles dans le lait algérien. A notre avis, cette deuxième hypothèse est à rejeter.

Nous avons, pour notre part, observé que la technique de culture sur milieu MRS en double couche avec incubation en aérobiose, comme l'a employée Harrati, était impropre parce que la croissance des streptocoques, trop nombreux, interdit tout développement aux lactobacilles. En revanche l'incubation en anaérobiose a donné des résultats satisfaisants; seuls les lactobacilles sont susceptibles de croître dans ces conditions.

L'évolution des levures et des moisissures est lente et régulière : leur développement tardif est dû à l'effet favorable de l'acidité développée dans le produit. Ces micro-organismes sont les seuls dont 
le nombre est plus important à la 44 heure, malgré l'adjonction d'eau qui a lieu entre ces deux moments. Il est probable que le barattage, qui provoque une aération très poussée du produit, active leur croissance.

Malgré leur nombre relativement faible par rapport à celui trouvé dans le Lben algérien (Harrati, 1974), le rôle de ces micro-organismes ne doit pas être négligeable; ils peuvent, d'une part, activer la croissance des ferments lactiques en libérant des facteurs de croissance dans le milieu, d'autre part, contribuer par leur métabolisme à donner au produit fini ses caractères organoleptiques spécifiques.

Le reste de la microflore étudiée, constitué par les streptocoques fécaux et les coliformes, détermine la qualité hygiénique du produit. Leur nombre est variable et ils ne semblent pas suivre une allure régulière, cependant, ils peuvent, respectivement, contribuer au processus d'acidification du produit, ou de production d'arôme.

\section{CONCLUSION}

Le Lben marocain est un lait fermenté utilisé surtout comme boisson rafraîchissante et apprécié pour ses qualités organoleptiques (acidité, arôme...), mais sa valeur nutritionnelle est loin d'être négligeable. En effet, il ne diffère du lait que par le léger mouillage dont il fait l'objet, par l'élimination d'une quantité variable de matière grasse, et par la fermentation d'une partie du lactose. Il est probable que la fraction azotée ne subit pas de modifications sensibles au plan nutritionnel, et que le développement microbien entraîne même un enrichissement en certaines vitamines. Il serait souhaitable qu'une étude précise soit effectuée visant à apprécier la valeur nutritionnelle de ce produit.

II serait également salutaire de fixer des normes physicochimiques et bactériologiques afin d'assurer au consommateur une qualité suffisante aux plans nutritionnel, organoleptique et hygiénique.

L'élaboration du Lben semble être liée en premier lieu au développement des streptocoques lactiques et des Leuconostoc (peu de lactobacilles ont été trouvés), avec, peut-être, un rôle accessoire de la flore fongique et de la flore d'origine fécale (coliformes et streptocoques fécaux). Nous envisageons, précisément, dans un travail ultérieur, de rechercher dans quelle mesure on pourrait, à l'aide de souches microbiennes sélectionnées provenant de nos échantillons de Lben, obtenir un produit d'assez bonne qualité organoleptique à partir d'une matière première pasteurisée. Ainsi pourraient être assurées en même temps une qualité hygiénique irréprochable et, éventuellement, une durée de conservation au froid suffisante pour permettre la distribution et la commercialisation du Lben obtenu dans des conditions économiquement acceptables. 


\section{Bibliographie}

Barnett (J. A.), Payne (R. W.) and Yarrow (D.) (1979). - A guide to identifying and classifying yeasts. Cambridge University Press, Cambridge.

BERGEY's (1974). - Manual of Determinative Bacteriology. Ed. the Williams and Wilkins Company, Baltimore, $8^{\mathrm{e}}$ édition.

Buttiaux (R.), Beerens (N.) et Tacquet (A.) (1974). - Manuel de techniques bactériologiques. Ed. Flammarion Médecine-Science, Paris, 4e édition.

F.I.L. (1962). - Détermination de la teneur en matière sèche du lait. Norme Internationale F.I.L.-I.D.F., 21.

Girard (H.) et Rougieux (R.) (1967). - Techniques de microbiologie agricole. Ed. Dunod, Paris.

Harrati (E.) (1974). - Recherches sur le Lben et le Klila algériens. Thèse de doctorat de spécialité, Université de Caen (France).

HaRrigan (W. F.) and Mc CANCE (Margaret E.) (1976). - Laboratory Methods in Food and dairy Microbiology. Academic Press, London, New York, San Francisco.

LODDER (J.) (1971). - The yeasts. A taxonomic study. North Holland Publishing Company, Amsterdam, London.

ShARPE (M. Elisabeth) (1979). - Identification of the Lactic Acid Bacteria. In "Identification Methods for Microbiology », vol. 1 (Ed. Skinner (F. A.) and Lovelock (D. W.), Academic Press, London, New York, Toronto, Sydney, San Francisco. 